\title{
Effect of Retrapping on Thermoluminescence Peak Intensities of Small Amorphous Silicon Quantum Dots
}

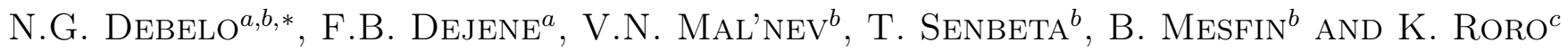 \\ ${ }^{a}$ Private Bag X13, Department of Physics, University of the Free State, QWA-QWA Campus, South Africa \\ ${ }^{b}$ Department of Physics, Addis Ababa University, P.O. Box 1176, Addis Ababa, Ethiopia \\ ${ }^{c} \mathrm{R} \& D$ Core (Energy Initiatives), Council for Scientific and Industrial Research, P.O. Box 395, Pretoria, South Africa
}

(Received October 24, 2015; in final form February 22, 2016)

The effect of retrapping on thermoluminescence intensity peak corresponding to each trap of small amorphous silicon quantum dots in three traps - one recombination center model is investigated. For first order kinetics, where there is no effect of retrapping, the thermoluminescence intensity clearly depends on the level of the trap beneath the edge of the conduction band. This energy difference between the edge of the conduction band and the level of the trap is called trap depth (activation energy). The shallowest trap gives the highest thermoluminescence intensity peak for first order kinetics. However, it was clearly observed that for second order and a case beyond second order kinetics, the thermoluminescence intensity peak corresponding to each trap does not depend on the trap depth. In this case, the retrapping probability coefficients are taken into account and most electrons which are detrapped from the shallow trap(s) will be retrapped to the deeper trap(s) resulting in fewer electrons taking part in the recombination process. This significantly reduces the thermoluminescence intensity peaks of the shallower trap(s). It was observed that the deepest trap, with very high concentration of electrons due to the retrapping phenomenon, gives the highest thermoluminescence intensity. In addition, the variation of concentration of electrons in each trap and the intensity of the thermoluminescence are presented. Though we considered the model of three traps and one recombination center, this phenomenon is true for any multiple traps.

DOI: 10.12693/APhysPolA.129.362

PACS/topics: 73.21.La, 73.63.Kv

\section{Introduction}

Electrons and holes created during the irradiation of a semiconductor will be trapped in some metastable states if they do not recombine rapidly and they need an energy which is equal to the trap depth to be released back to the conduction band to give luminescence. This energy is also called activation energy. If the detrapping process is caused by heating or thermostimulation, the luminescence is called thermoluminescence (TL) [1-5].

Traps and trapped carriers in nanoparticles are abundant, and luminescence might be improved if the trapped carriers are effectively released from the traps by stimulation with energy equal to the trap depth. TL is a good way to detect the recombination emission caused by detrapping of carriers thermally [5]. TL continues to be an active area of research because of its immense contribution in the fields of personal and environmental dosimetry, dating of archaeological artifacts, sediments and study of defects in solids [1]. In particular, light emission from silicon quantum dots has got recent attention because of its potential applications in the silicon-based optoelectronic devices [6-10]. There are different mechanisms of increasing the efficiency of the light emitting device. In experiments, the possible increase in the intensity of TL from nanoparticles due to the confinement effect and increase in the surface states have been studied [11]. It was shown that both increase the probability of radiative recombination and enhance the intensity of the TL.

The TL from different materials has been studied by many research groups using different models [12-14].
The simplest possible model that has been used to describe the process by which materials emit light when heated consists of two localized levels: an isolated electron trap and a recombination center. This approach is commonly called one-trap-one-recombination center (OTOR) model [13].

The interactive-multi-trap-system (IMTS) model has also been used [14]. It assumes that there are one active electron trap, one thermally disconnected deep trap that cannot be thermally activated, and one recombination center.

In OTOR model, the peak of the TL intensity corresponding to each peak depends on the level of the trap beneath the edge of the conduction band; the shallower the trap, the higher the intensity peak. In other words, retrapping has no effect on the intensity peaks. In IMTS model, though there is an effect of retrapping to the thermally disconnected deep trap, its effect on TL peaks is not clearly observed as there is only one active electron trap taking part in the TL process. Basically, in reality we often have more than one active electron traps taking part in TL process and the intensity peaks completely depend on the retrapping condition in this case.

In this paper we consider small a-Si quantum dots of different size with size dependent radiative recombination rate and study the effect of retrapping on TL intensity peaks corresponding to each trap for second order and a case beyond second order kinetics assuming three active electron traps and one recombination center model. In addition, we study the variation of concentration of electrons in each trap and the intensity of the TL. 


\section{Statement of the problem}

Consider the following energy band scheme having four localized levels (Fig. 1). The three levels act as traps $\left(T_{1}, T_{2}\right.$, and $\left.T_{3}\right)$ and the other acts as recombination center $(R)$. The absorption of radiation of energy greater than the band gap energy results in the ionization of valence electrons, producing free electrons in the conduction band and free holes in the valence band, respectively (transition 1).

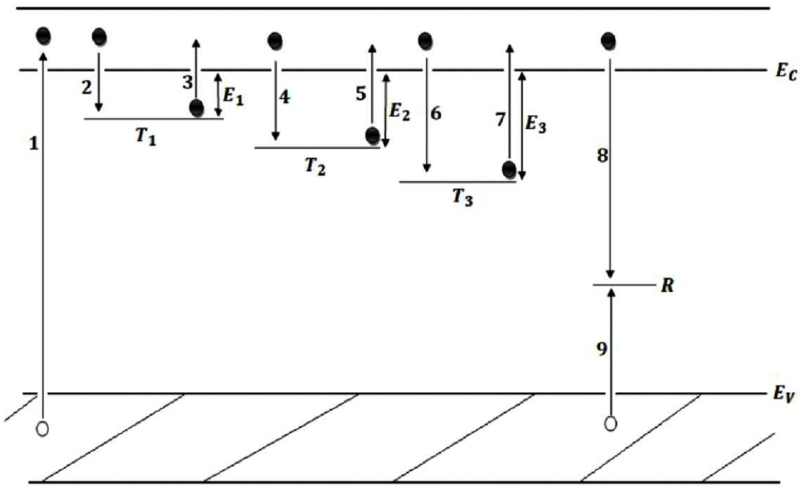

Fig. 1. Four level model for thermoluminescence. Allowed transitions: (1) ionization; (2), (4), (6) and (9) trapping; (3), (5) and (7) thermal release; (8) radiative recombination and emission of light.

The electrons and holes may either recombine with each other or become trapped at the trap centers. In order for recombination to occur holes first become trapped at centers $(R)$ (transition 9 ). Recombination takes place via the annihilation of the trapped holes by free electrons (transition 8). If the recombination transition is assumed to be radiative, then luminescence will result. The free electrons may also become trapped at levels $T_{1}, T_{2}$ and $T_{3}$ (transitions 2, 4 and 6 ) in which case recombination can only take place if the trapped electrons absorb enough energy $E_{1}, E_{2}$, and $E_{3}$ respectively to be released back to conduction band (transitions 3,5 and 7 ), from where recombination is possible. In other words, the TL occurs when the trapped electrons absorb enough energy in the form of heat to be released back to the conduction band from where there is possible recombination of electrons and holes. The energy required for the trapped electrons to be released back to the conduction band is called the trap depth (activation energy). Next we write down the rate equations governing the TL process.

\section{Rate equations}

Let us consider three active electron traps with energy levels $E_{1}, E_{2}$, and $E_{3}$ below the bottom of the conduction band. The rate equations can be written as

$$
\frac{\mathrm{d} n_{1}}{\mathrm{~d} t}=-s_{1} n_{1} \exp \left(-\frac{E_{1}}{k T}\right)+n_{\mathrm{c}} A_{1}\left(N_{1}-n_{1}\right)
$$

$$
\begin{aligned}
& \frac{\mathrm{d} n_{2}}{\mathrm{~d} t}=-s_{2} n_{2} \exp \left(-\frac{E_{2}}{k T}\right)+n_{\mathrm{c}} A_{2}\left(N_{2}-n_{2}\right), \\
& \frac{\mathrm{d} n_{3}}{\mathrm{~d} t}=-s_{3} n_{3} \exp \left(-\frac{E_{3}}{k T}\right)+n_{\mathrm{c}} A_{3}\left(N_{3}-n_{3}\right), \\
& \frac{\mathrm{d} n_{\mathrm{c}}}{\mathrm{d} t}=s_{1} n_{1} \exp \left(-\frac{E_{1}}{k T}\right)+s_{2} n_{2} \exp \left(-\frac{E_{2}}{k T}\right) \\
& +s_{3} n_{3} \exp \left(-\frac{E_{3}}{k T}\right)-n_{\mathrm{c}}\left[A_{1}\left(N_{1}-n_{1}\right)\right. \\
& \left.\quad+A_{2}\left(N_{2}-n_{2}\right)+A_{3}\left(N_{3}-n_{3}\right)+A_{\mathrm{r}} n_{\mathrm{h}}\right] .
\end{aligned}
$$

Here $n_{i}(i=1,2,3)$ is the concentration of trapped electrons $\left[\mathrm{cm}^{-3}\right]$ on the levels $E_{i}[\mathrm{eV}], N_{i}(i=1,2,3)$ is the total concentration of traps $\left[\mathrm{cm}^{-3}\right]$ on the levels $E_{i}[\mathrm{eV}]$, $k$ is the Boltzmann constant $\left[\mathrm{JK}^{-1}\right] ; s_{i}(i=1,2,3)$ is the probability of electron to escape from the $i$-th trap $\left[\mathrm{s}^{-1}\right]$, $A_{i}(i=1,2,3)$ is the retrapping probability on the $i$-th energy level $\left[\mathrm{cm}^{3} \mathrm{~s}^{-1}\right] ; n_{\mathrm{c}}$ and $n_{\mathrm{h}}$, respectively, are the concentration of the electrons in the conduction band and holes in the hole trap $\left[\mathrm{cm}^{-3}\right]$, and $A_{\mathrm{r}}$ is the electron-hole radiative recombination probability $\left[\mathrm{cm}^{3} \mathrm{~s}^{-1}\right]$. It is assumed that, initially, all holes are trapped to the hole trap. The term $s$ is commonly called the "frequency factor", although when applied to TL it is often called "attempt-to-escape frequency". The usual interpretation of $s$ is that it represents the number of times per second that a bound electron interacts with lattice phonons times transition probability [1]. The maximum value expected for $s$ is therefore the lattice vibration frequency, namely $10^{12}-10^{14} \mathrm{~s}^{-1}$. The system (1)-(4) is closed by the quasi-neutrality condition

$$
n_{1}(T)+n_{2}(T)+n_{3}(T)+n_{\mathrm{c}}(T)=n_{\mathrm{h}}(T) .
$$

Here $n_{\mathrm{h}}(T)$ is the temperature dependent concentration of holes in hole trap. Basically the temperature of the system depends on time $T=T(t)$. The generally acceptable is the linear heating rate

$$
T(t)=T_{0}+\beta t,
$$

where $\beta$ is the heating rate. The system of Eqs. (1) (6) presents the rate equations governing the process of TL with the possibility of multiple retrapping. It is the recombination term $A_{\mathrm{r}} n_{\mathrm{h}}(T) n_{\mathrm{c}}(T)$ in Eq. (4) which determines the profile of the TL curve. Thus the intensity of the TL is given by

$$
I(T)=A_{\mathrm{r}} n_{\mathrm{h}}(T) n_{\mathrm{c}}(T) .
$$

The generalization of the system of Eqs. (1)-(4) for a greater number of traps is trivial. The above presented system of equations is nonlinear with varying coefficients. In Eqs. (1)-(4), the variables $A_{1}, A_{2}, A_{3}$, and $A_{\mathrm{r}}$ are parameters of amorphous silicon quantum dots.

\section{First order kinetics}

In first order kinetics, the assumption is that there is a strong tendency to recombination and that electrons, which are released thermally from the traps and excited into the conduction band recombine quickly with trapped holes. Hence the retrapping probability coefficients $A_{i}(i=1,2,3)$ can be neglected and Eqs. (1)-(4) 
are analytically solvable. In this case, the variation of concentration of electrons in each trap is given by

$$
n_{i}(T)=n_{i 0} \exp \left(-\left(\frac{s_{i}}{\beta}\right) \int_{T 0}^{T} \exp \left(-\frac{E_{i}}{k T}\right) \mathrm{d} T\right) .
$$

The contribution of each trap to the TL intensity $I_{i}(T)(i=1,2,3)$ is given by

$$
\begin{aligned}
& I_{i}(T)=n_{i 0} s_{i} \exp \left(\frac{-E_{i}}{k T}\right) \\
& \quad \times \exp \left(-\left(\frac{s_{i}}{\beta}\right) \int_{T 0}^{T} \exp \left(\frac{-E_{i}}{k T}\right) \mathrm{d} T\right),
\end{aligned}
$$

where $n_{i 0}$ is the initial concentration of electrons at $T=0$.

\section{Second order and a case beyond second order kinetics}

The consideration of retrapping terms with the coefficients $A_{1}, A_{2}$, and $A_{3}$ considerably complicates the system of Eqs. (1)-(4) that becomes nonlinear and cannot be solved analytically in general case. We solved this system of equations numerically with the help of Mathematica 8 with the parameters

$$
\begin{aligned}
& E_{1}=0.65 \mathrm{eV}, \quad E_{2}=0.8 \mathrm{eV}, \quad E_{3}=1 \mathrm{eV}, \\
& n_{1}(0)=n_{2}(0)=n_{3}(0)=10^{15} \mathrm{~cm}^{-3}, \quad \beta=1^{\circ} \mathrm{C} / \mathrm{s}, \\
& s_{1}=s_{2}=s_{3}=10^{8} \mathrm{~s}^{-1}, \\
& N_{1}=N_{2}=N_{3}=10^{20} \mathrm{~cm}^{-3} .
\end{aligned}
$$

For the typical quantum dots the retrapping probability is of the order of $10^{7} \mathrm{~cm}^{3} \mathrm{~s}^{-1}$. We chose $A_{1}=A_{2}=$ $A_{3}=5 \times 10^{7} \mathrm{~cm}^{3} \mathrm{~s}^{-1}$ and ignore the possible corrections associated with the confinement.

The size dependent radiative recombination probability for amorphous silicon (a-Si) quantum dots of radii $1-3 \mathrm{~nm}$ is theoretically calculated [5] and summarized in Table I. We adopt this size dependence of radiative recombination probability for our model.

\section{TABLE I}

Approximate values of radiative recombination rate calculated for a-si quantum dots.

\begin{tabular}{c|c}
\hline \hline$R[\mathrm{~nm}]$ & $A_{\mathrm{R}}\left[\mathrm{cm}^{3} \mathrm{~s}^{-1}\right]$ \\
\hline 2 & $2 \times 10^{7}$ \\
4 & $3 \times 10^{6}$ \\
6 & $4 \times 10^{5}$
\end{tabular}

\section{Results and discussion}

Figure 2 shows the variation of the concentration of electrons in the traps for first order kinetics. It is clearly seen that there is no effect of retrapping and the traps

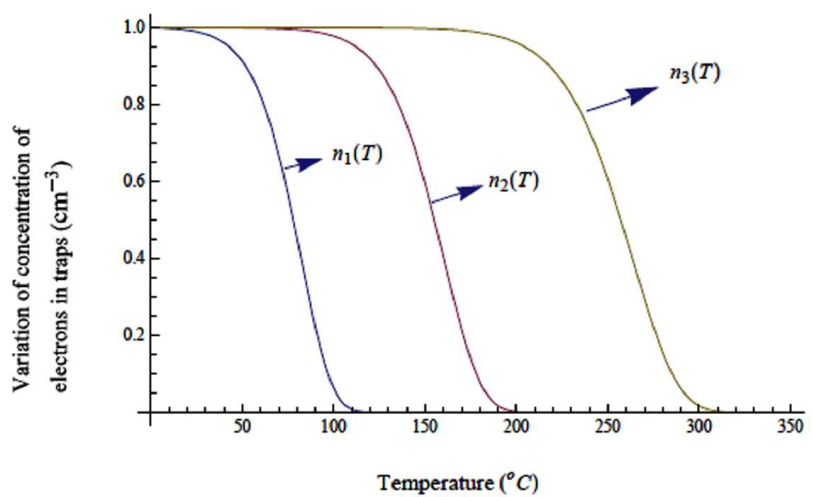

Fig. 2. Variation of concentration of electrons in traps (first order kinetics).

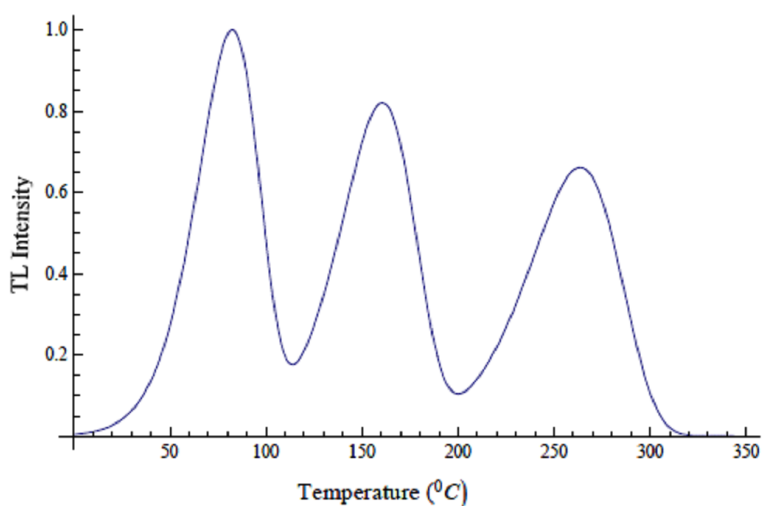

Fig. 3. TL intensity $I(T)$ versus temperature (first order kinetics).

are activated at different temperatures. The initial concentration of electrons in each trap is assumed to be the same.

Figure 3 shows the TL intensity versus temperature for first order kinetics. It is clearly observed that the TL peaks depend on the trap depth. The shallowest trap gives the highest TL peak and the deepest trap gives the lowest TL peak in first order kinetics.

Figures 4, 5 and 6 show the variation of the concentration of electrons in the traps with activation energies $E_{1}=0.65 \mathrm{eV}, E_{2}=0.8 \mathrm{eV}$ and $E_{3}=1 \mathrm{eV}$, respectively, for quantum dots of different radii $R$ from $1-3 \mathrm{~nm}$. Here, the effect of retrapping clearly comes in. The electrons that are released from the first trap level $\left(E_{1}=0.65 \mathrm{eV}\right)$ can be retrapped by the deeper traps $\left(E_{2}=0.8 \mathrm{eV}\right.$ and $\left.E_{3}=1 \mathrm{eV}\right)$. It explains an increase in the concentration of electrons in the deeper traps with temperature as indicated in Figs. 4 and 5. One can see from Figs. 4-6 that $n_{1}(T), n_{2}(T)$ and $n_{3}(T)$ depend on the size of quantum dot as well.

It is interesting to see how the concentration of trapped electrons $n_{1}(T), n_{2}(T)$, and $n_{3}(T)$ vary with temperature and size. This will not be obtained from experimental results in which only the TL glow curves are generated. 


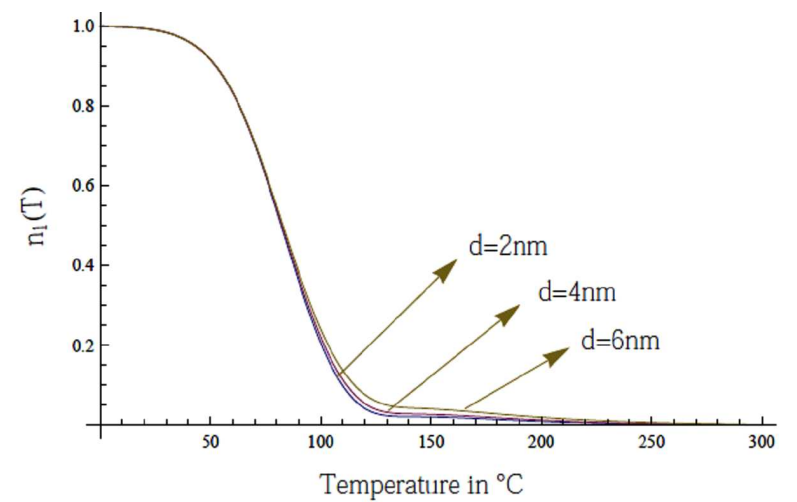

Fig. 4. Numerical simulation of variation of concentration of electrons $n_{1}(T)$ in the shallower trap $\left(E_{1}=\right.$ $0.65 \mathrm{eV})$.

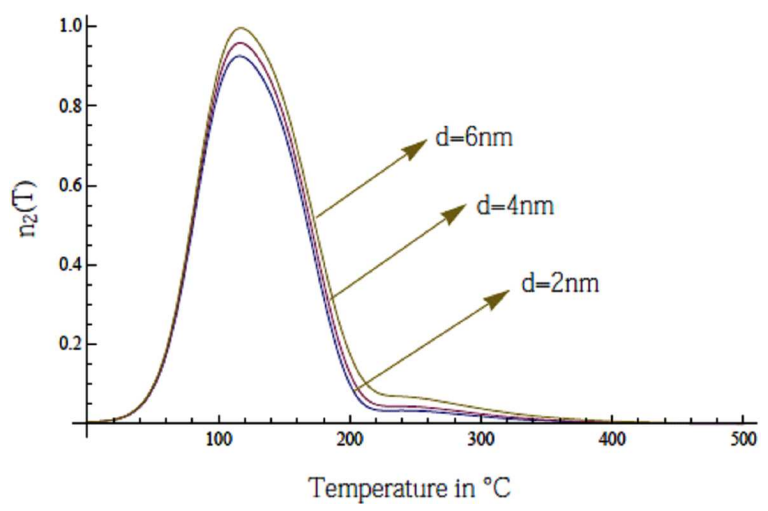

Fig. 5. Numerical simulation of variation of concentration of electrons $n_{2}(T)$ in the deeper trap $\left(E_{2}=0.8 \mathrm{eV}\right)$.

Table I shows that the number of the carriers, which are recombined radiatively per unit time, increases with a decrease in the quantum dot size. Using the data of Table I and Eq. (7), we simulated the temperature dependent TL intensity for the dot whose radius varies in the range $1-3 \mathrm{~nm}$ as presented in Fig. 7 . The intensity increases with decreasing in the dot size indicating that

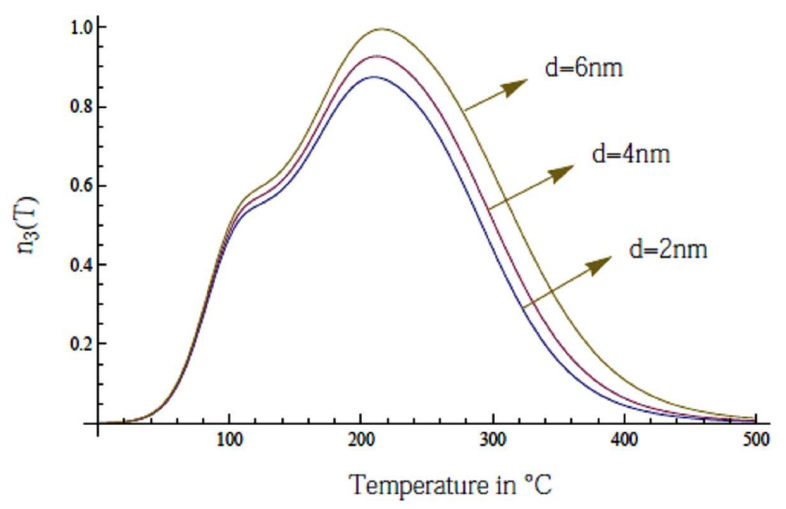

Fig. 6. Numerical simulation of variation of concentration of electrons $n_{3}(T)$ in the deepest trap $\left(E_{3}=1 \mathrm{eV}\right)$. the quantum confinement effect enhances band to band radiative recombination rate. There are three peaks of the TL intensity and they correspond to the three different active electron trap levels. The deepest trap gives the highest TL intensity which means that the TL intensity does not depend on the trap depth for second order and a case beyond second order kinetics for the case of multiple traps and one recombination center. The TL intensity is monitored by the effect of retrapping. We also found that positions of the TL intensity peaks corresponding to the first and second traps practically do not shift with the temperature. At the same time, the intensity peaks of the third trap slightly shift to the high temperatures with increase in the dot size. The TL peaks of the first and second traps are lower comparing with the peaks of the third trap (Fig. 7). It can be explained by the fact that during the excitation of the first and the second traps, only some part of its electrons recombine with the holes emitting light. Another part of them are trapped by the third trap. This is indicated in the initial rise in the concentration of the trapped electrons in the third trap (Fig. 6).

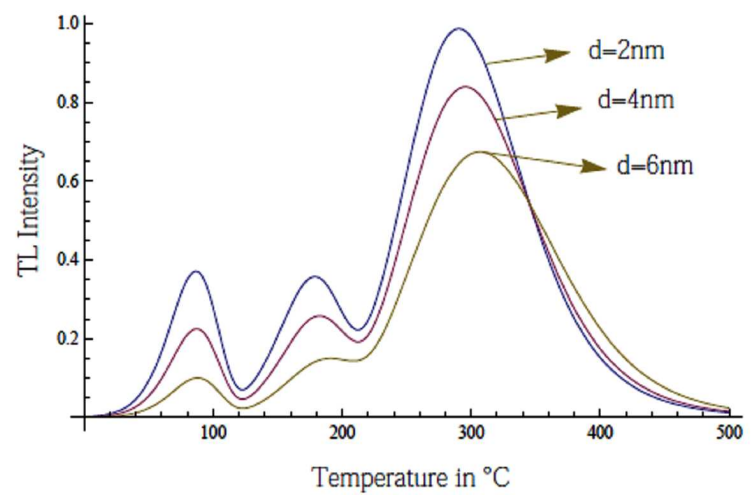

Fig. 7. Numerical simulation of TL intensity I versus temperature.

The other interesting part is that experimental results for any TL material generate only the TL glow curves and give no result concerning the variation of concentration of electrons in traps. However, this model generates the variation of concentration of trapped electrons in different traps of different trap depths below the edge of the conduction band with temperature.

Negative photoconductivity, optical and thermal quenching effects can be observed even with two level model. Particularly, thermal quenching effect is the most common phenomenon in thermoluminescence. However, this effect usually becomes significant at higher heating rates. In our simulation work we considered very small heating rate which is $1^{\circ} \mathrm{C} / \mathrm{s}$ so that thermal quenching effect could be minimized.

\section{Conclusion}

The effect of retrapping on the TL glow curves of small a-Si quantum dots of radii $1-3 \mathrm{~nm}$ in the model of three 
active electron traps and one recombination center has been numerically simulated. There are three peaks of the TL intensity corresponding to the three active electron trap levels. Our numerical results show that the TL intensity increases with decrease in the quantum dot size. This can be explained in terms of the quantum confinement effect. In this paper, we account it with the help of extrapolation of the theoretically calculated data on the probability of radiative transitions on the quantum dot size. We obtained that the third trap with trap level $E_{3}=1 \mathrm{eV}$ provides the highest TL intensity as compared with the first trap $\left(E_{1}=0.65 \mathrm{eV}\right)$ and the second trap $\left(E_{2}=0.8 \mathrm{eV}\right)$ at higher temperature because of its high trapped electron concentration. The point is that the majority of the electrons released by the first and the second traps will be retrapped by the third one. All the traps show increase in the TL intensities with decrease in the quantum dots size. To our mind the ensemble of quantum dots of different sizes with different active electron trap levels may have interesting TL properties and deserves further study.

\section{Acknowledgments}

Dedicated to Prof. Vadim Nicholavich Mal'nev; the late professor of physics at the Department of Physics of Taras Shevchenko Kyiv National University and Addis Ababa University.

\section{References}

[1] S.W.S. Mckeever, Thermoluminescence of Solids, Cambridge Solid State Science Series, Oklahoma State University, Cambridge, 1988, p. 1.

[2] V. Pagonis, G. Kitis, C. Furetta, Numerical and Practical Exercises in Thermoluminescence, Springer, New York, 2005, p. 80.
[3] Yu.A. Skryshevskii, V.A. Skryshevskii, J. Appl. Phys.89, 2711 (2001).

[4] C. Furetta, Handbook of Thermoluminescence, Cambridge University Press, 1988, p. 1.

[5] N.M. Abdul-Ameer, M.C. Abdulrida, J. Mod. Phys.2, 1530 (2011).

[6] K. Dohnalova, T. Gregorkiewicz, K. Kusova, J. Phys. Condens. Matter 26, 173201 (2014).

[7] S. Martini, L. Khl Teles, M. Marques, A.E.B. Marques, A.A. Quivy, World J. Condens. Matter Phys. 1, 161 (2011)

[8] Eun-Chel Cho, M.A. Green, G. Conibeer, D. Song, Y.-H. Cho, G. Scardera, S. Huang, S. Park, X.J. Hao, Y. Huang, L. Van Dao, Advances in OptoElectronics, Hindawi Publishing Corporation, Cairo, 2007, p. 1.

[9] N.G. Debelo, S.K. Ghoshal, Lat. Am. J. Phys. Educ. 5, 428 (2011).

[10] A. Antipov, M. Bell, M. Yasar, V. Mitin, W. Scharmach, M. Swihart, A. Verevkin, A. Sergeev, Nanoscale Res. Lett. 6, 142 (2011); Indones. J. Phys. 22, 125 (2011).

[11] D. Mendoza-Anaya, C. Angeles, P. Salas, R. Rodriguez, V.M. Castano, Nanotechnology 14, 19 (2003).

[12] L. Robindro Singh, S. Dorendrajit Singh, J. Nanomater. 2012, 239182 (2012).

[13] V. Pagonis, G. Kitis, Phys. Status Solidi B249, 1 (2012).

[14] I. Sychugov, Synthesis and Properties of Single Luminescent Silicon Quantum Dots (Doctoral thesis), Royal Institute of Technology, Sweden, (2006). 